\title{
Recurrent Rapenzul Syndrome in a Male Child; A Rare Surgical Entity
}

Nand Lal Kella, Mumtaz Ahmed Qureshi, Imtiaz Ahmed Qureshi

\section{ABSTRACT}

Rapunzel syndrome is a rare entity when the undigested tuft of hairs accumulated in the stomach and extends beyond the pylorus in the small intestine with a presentation of vomiting, pain in epigastrium and a palpable mass. Recurrence of this pathology is extremely rare, and upto now, only six cases have been reported. We report a male who was partially deaf and dumb, presented with trichophagia and trichobezoar in the age of 5 and 9 years. Both times gastrostomy was performed and trichobezoars were removed completely which were extending in the small intestine. Post-operative recovery was un-eventful and the patient has been referred to the psychiatrist for further management.

KEY WORDS: Trichobezoar, Psychiatric, Rapunzel syndrome, male child

This article may be cited as: Kella NL, Qureshi MA, Qureshi IA. Recurrent Rapenzul Syndrome in a Male Child; A Rare Surgical Entity. J Liaquat Uni Med Health Sci. 2018;17(02):106-8. doi: 10.22442 jlumhs. 181720560

\section{INTRODUCTION}

Rapunzel syndrome (RS) is a rare surgical condition, it occurs when gastric trichobezoar extends beyond the pylorus and may reach to the small intestine but occasionally reaches up to the colon ${ }^{1}$. Rapunzel syndrome was first described by Vanghan in 1968, and it is named after the long- haired 12 years old princess Rapunzel imprisoned (Captive) by a witch in the castle. It is a German fairy tell written by two brothers Jacob and Wilhelm in $1812^{2}$.

Rapunzel syndrome is an uncommon problem in children and most commonly affected females' ranges from 20-40 years. Till now 88 cases of Rapunzel syndrome has been searched in literature till the end of $2016^{3}$.

Recurrent Rapunzel syndrome is an extremely rare pathology in children, so for only six cases have been reported, all the affected children were girls ${ }^{4}$. Memon reported the first recurrent Rapunzel syndrome in twelve years old girl ${ }^{5}$. We present an additional first male recurrent Rapunzel syndrome.

\section{CASE REPORT}

A 5 Year old male child was first admitted in June 2013, with vomiting, decrease intake and pain in epigastrium for last few months. History favors that child was too much eager to pick hairs from beds, floor and even on roads, but never plucks/eats his own hair. Clinical examination showed a non-tender hard big palpable mass extending from the left hypochondrium to epigastrium. Child was anemic with normal vitals. He had, hearing and talking issues. $\mathrm{He}$ can partially listen but unable to talk properly and do work on commands. If anybody does not obey him he becomes aggressive and beat himself and parents also.

Blood Investigations; Serum electrolytes, urea, creatinine, amylase and liver function tests were within normal limits, while complete blood count showed low hemoglobin level and slightly raised total leukocytes count. X-ray abdomen erect posture was insignificant with few calcifications in left hypochondria. Ultrasonography revealed a $10 \mathrm{~cm}$ echogenic mass casting posterior acoustic shadow seen within bowel loops in epigastric region with calcifications. Barium study showed a large filling defect in stomach suggestive of trichobezoar (Fig I). After blood transfusion, surgery was planned and gastrostomy did, a long trichobezoar removed acquiring the shape of the stomach and extending up to the jejunum/ileum approximately length was around $70 \mathrm{~cm}$ (Fig II). Stomach was closed in two layers. Patient was discharged without any problem on 6th post-operative day. The Patient was referred to a psychiatrist for evaluation and treatment. After surgery, he consulted me up to two months then having no communication.

FIGURE I: BARIUM MEAL IMAGE SHOWING FILLING DEFECT

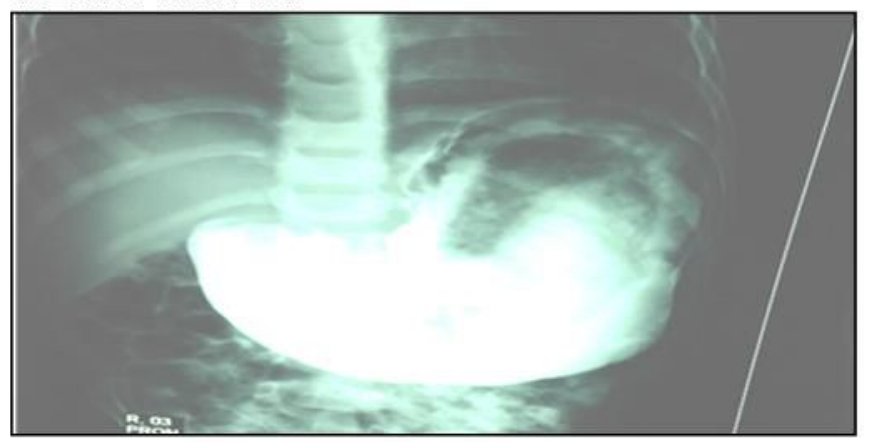




FIGURE II:
PHOTOGRAPH SHOWING
TRICHOBEZOAR BEING
EXTRACTED DURING
FIRST LAPAROTOMY

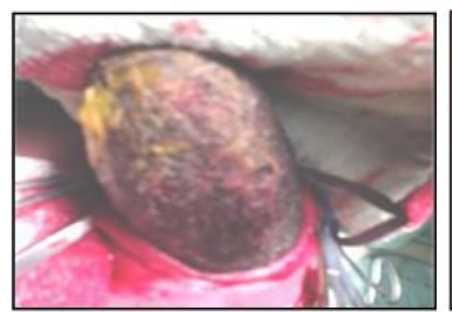

trapped in gastric rugeas and get accumulated in the form of mass getting a shape of the stomach when it extends beyond the pylorus it is supposed to be as Rapunzel syndrome ${ }^{4}$.

About $90 \%$ of cases of trichobezoar are found in females with age ranges from 13-19 years with undiscovered psychiatric problem ${ }^{6}$. Gupta in his series showed that $37.5 \%$ patients were male with youngest one of four years ${ }^{2}$. Our patient was male with five years age.

The psychological disorder associated with this entity is trichotillomania (plucking of hairs) which was described in the literature in 1889 as an impulse control disorder according to the diagnostic statistic manual DSM-IV). About $30 \%$ of trichotillomania patient having trichophagia and $50 \%$ patient having trichophagia leading to trichobezoar ${ }^{2,4}$. Our patient had strong history of trichophagia but having no trichotillomania. Recurrent Rapunzel syndrome (RRS) is the extremely rare entity. Till now only six cases of RRS have been reported, three under the age of 12 years and remaining three above the age of fifteen years ${ }^{4}$. All affected patients were females, while our patient was the first male with recurrence.

Recurrent Rapunzel syndrome patients usually present with pain in abdomen, nausea and vomiting, weight loss, anemia and a palpable mass in epigastri$u^{5}$. If left untreated may lead to complications; intestinal perforation, pancreatitis and acute intestinal obstruction ${ }^{7}$. Our patient presented at the age of 5 and 9 years with complaints of pain in abdomen, vomiting and weight loss and trichophagia. Majority of patients with Rapunzel syndrome present late while a high index of suspicion should be made with trichophagia, vomiting, pain in abdomen and a palpable mass in epigastrium.

X-Ray plain of the abdomen is not helpful, but may give an appearance of mottled masses, outlined by gases in the distended stomach ${ }^{2}$. Upper Gl contrast reveals a large intraluminal filling defect with extension in small bowel in the case of Rapunzel syndrome ${ }^{4}$. Classical honeycomb appearance is seen when barium becomes trapped in the interstices of bezoar. In the first presentation, our patient had the classical appearance of honey comb on upper Gl contrast, while second- time ultrasonography was suggestive of the trichobezoar.

Ultrasonography has the diagnostic efficacy of $80 \%$, while CT scan has $97 \%$ sensitivity for trichobezoar ${ }^{7,8}$. CT scan is highly suggestive of trichobezoar ${ }^{9}$ while endoscopy is suggested as gold standard for diagnostic as well as therapeutic for small bezoar ${ }^{5}$.

Successful treatment and good prognosis of the trichobezoar is removal of the mass and prevention of recurrence by treating the associated psychological

Trichobezoars are formed as a result of the accumulation of hairs in the stomach. Hairs being slippery 
illness. Treatment options for Psychological illnessare, anti-psychotic drugs, psychotherapy, and change of atmosphere. This can be done by parents and multidisciplinary team approach ${ }^{2,10}$

Treatment options include endoscopy removal, chemical dissolution, mechanical fragmentation and laser ignition, they are helpful for small bezoars? Gorter $^{11}$ in his study of 108 cases had shown that laparotomy was $100 \%$ successful, while laparoscopy $75 \%$ and endoscopy had $5 \%$ success rate. We had done laparotomy both times because of big size and expected adhesion for the second time.

\section{CONCLUSION}

Recurrent Rapunzel syndrome is an extremely rare entity, may be anticipated in the patient having improper attention for psychological treatment. A multi -disciplinary team approach for previously diagnosed trichobezoar can prevent the relapse of this entity.

\section{REFERENCES}

1. Tiwary SK, Kumar S, Khanna R, Khanna AK. Recurrent Rapunzel syndrome. Singapore Med J. 2011; 52(6):e128-130

2. Gupta R, Prabhakar G, Mathur P, Goyal RB, Sharma C, Ali MA. Rapunzel syndrome and its variant in pediatric patients: Our experience. Arch Int Surg 2014; 4(3): 152-7

3. Ullah W, Saleem K, Ahmed E, Anwer F. Rapunzil syndrome: a rare cause of hypoproteinemia and review of literature. BMJ Case Rep. 2016; pii: bcr2016216600. doi: 10.1136/bcr-2016-216600

4. Obinwa O, Cooper D, Khan F, O'Riordan JM.
Rapunzel syndrome is not just a mere surgical problem: A case report and review of current management. World J Clin Cases 2017; 5(2): 5055. doi: $10.12998 / w j c c . v 5.12 .50$

5. Memon SA, Mandhan P, Qureshi JN, Shairani A. Recurrent rapunzil syndrome -a case report. Med Sci Monit 2003; 9:92-4

6. Kajal P, Bhutani N, Tyagi N, Arya P. Trichobezoar with and without Rapunzel syndrome in pediatric population: A case series from a tertiary care Centre of Northern India. Int J Surg Case Rep 2017; 40: 23-6. doi: 10.1016/j.ijscr.2017.08.060

7. Ahmed N, Baloch MA, Baber KM, Ahmed J. A rare variant of Rapunzel syndrome-acute small bowel obstruction caused by ball of hairs in distal ileum with its tail extending in the caecum and ascending colon. J Pak Med Assoc. 2016; 66(6): $761-4$

8. Mir A. Trichobezoar. J Coll Physicians Surg Pak 2011; 21: 763-5

9. Wang $Z$, Coa F, Liu D, Fang Y, Li F. The diagnosis and treatment of Rapunzel syndrome. Acta Radiol Open 2016; 5(11): 1-4

10. Jones GC, Continho K, Anjaria D, Hussain N, Dholakia R. Treatment of recurrent Rapunzel syndromeTrichotillomania, Case report and literature review. Psychosomatics 2010; 51(5): 443-6. doi: 10.1176/appi.psy.51.5.443.

11. Gorter RR, Kneepkens CM, Mattens EC, Aronson DC, Heij HA. Management of trichobezoar: case report and literature review. Pediatr Surg Int 2010; 26(5): 457-63. doi: 10.1007/s00383-010-2570-0.

\begin{tabular}{|l|}
\multicolumn{1}{c|}{ AUTHOR AFFILLATION: } \\
Prof. Nand Lal Kella (Corresponding Author) \\
Department of Pediatric Surgery \\
Liaquat University of Medical and Health Sciences \\
(LUMHS), Jamshoro, Sindh-Pakistan. \\
Email nckella@gmail.com \\
Dr. Mumtaz Ahmed Qureshi \\
Assistant Professor \\
Department of Pediatric Surgery \\
LUMHS, Jamshoro, Sindh-Pakistan. \\
Dr. Imtiaz Ahmed Qureshi \\
Assistant Professor \\
Department of Pediatric Surgery \\
LUMHS, Jamshoro, Sindh-Pakistan.
\end{tabular}

